\title{
Interstitial carbon in bcc HfNbTiVZr high-entropy alloy from first principles
}

\author{
Luis Casillas-Trujillo $\odot,{ }^{1,{ }^{*}}$ Ulf Jansson, ${ }^{2}$ Martin Sahlberg, ${ }^{2}$ Gustav Ek, ${ }^{2}$ Magnus M. Nygård $\odot,{ }^{3}$ Magnus H. Sørby $\odot,{ }^{3}$ \\ Bjørn C. Hauback $\odot,{ }^{3}$ Igor A. Abrikosov $\odot,{ }^{1,4}$ and Björn Alling ${ }^{1}$ \\ ${ }^{1}$ Department of Physics, Chemistry and Biology (IFM), Linköping University, 58183 Linköping, Sweden \\ ${ }^{2}$ Department of Chemistry-Ångström, Uppsala University, 75121 Uppsala, Sweden \\ ${ }^{3}$ Department for Neutron Materials Characterization, Institute for Energy Technology, NO-2027 Kjeller, Norway \\ ${ }^{4}$ Materials Modeling and Development Laboratory, National University of Science and Technology “MISIS,” Moscow 119049, Russia
}

(Received 7 October 2020; accepted 11 November 2020; published 2 December 2020)

\begin{abstract}
The remarkable mechanical properties of high-entropy alloys can be further improved by interstitial alloying. In this work we employ density functional theory calculations to study the solution energies of dilute carbon interstitial atoms in tetrahedral and octahedral sites in bcc HfNbTiVZr. Our results indicate that carbon interstitials in tetrahedral sites are unstable, and the preferred octahedral sites present a large spread in the energy of solution. The inclusion of carbon interstitials induces large structural relaxations with long-range effects. The effect of local chemical environment on the energy of solution is investigated by performing a local cluster expansion including studies of its correlation with the carbon atomic Voronoi volume. However, the spread in solution energetics cannot be explained with a local environment analysis only pointing towards a complex, long-range influence of interstitial carbon in this alloy.
\end{abstract}

DOI: 10.1103/PhysRevMaterials.4.123601

\section{INTRODUCTION}

In recent years high-entropy alloys (HEAs) have gathered vast interest due to their mechanical properties. In particular, HEAs consisting of refractory elements have possible applications as high performance materials at elevated temperatures. Near equimolar WTaMoNb and WTaMoNbV have displayed better mechanical properties at elevated temperatures than conventional $\mathrm{Ni}$ based superalloys [1]. Besides mechanical properties, HEAs have been investigated as highly tailorable hydrogen storage materials [2,3]. HfNbTiVZr has attracted special attention for its excellent hydrogen storage capacity [4], with experimental data suggesting that hydrogen can occupy both tetrahedral and octahedral positions in the face centered structured (fcc) phase [5,6]. Furthermore, $\mathrm{Hf}_{21} \mathrm{Nb}_{25} \mathrm{Ti}_{15} \mathrm{~V}_{15} \mathrm{Zr}_{24}$ has also been reported to display superconductivity [7]. HfNbTiVZr is believed to be a single-phase HEA with a body centered cubic (bcc) structure thermodynamically stable above $800^{\circ} \mathrm{C}$. At lower temperatures the thermodynamically stable phase is a mixture of bcc and hexagonal closed packed (hcp) alloys and a Laves phase [8]. However, metastable solid solution samples have been obtained at room temperature $[9,10]$.

The properties of refractory HEAs can be further improved by interstitial solution of other elements in the structure. For example, the incorporation of carbon atoms into HEAs has

\footnotetext{
*luis.casillas.trujillo@liu.se
}

Published by the American Physical Society under the terms of the Creative Commons Attribution 4.0 International license. Further distribution of this work must maintain attribution to the author(s) and the published article's title, journal citation, and DOI. Funded by Bibsam. been studied experimentally [11-23], displaying an increase in yield and ultimate strengths. To understand the change in mechanical properties from the incorporation of carbon, an atomic scale understanding is needed. As such, ab initio based studies are a powerful tool and a key piece to develop strategies to fine-tune mechanical properties. Recently, Ikeda et al. [24] have studied the impact of interstitial carbon in fcc structured $\mathrm{CrMnFeCoNi}$ using first principles calculations. Carbon atoms prefer to occupy octahedral interstitial sites in bcc metals such as $\mathrm{Fe}[25,26], \mathrm{Nb}$, and $\mathrm{V}$. The small size of the octahedral interstice produces a low maximum solid solubility of carbon in pure metals in group 4-6 of the Periodic Table, and therefore a low carbon solubility in solid bcc HEA would also be expected. However, carbon dissolution could be assisted by the different interstitial site sizes, produced by lattice distortions originating from the atomic size mismatch, in addition to the effect of local chemical environments. We address these research questions in the present work. The constituent elements in HfNbTiVZr possess a large variation in atomic radii, allowing us to investigate the influence of metals with different radii on the size distribution of interstitial sites and how this size distribution and chemical bonding of an interstitial atom are correlated. We also study the further distortions produced by the incorporation of carbon. Using density functional theory (DFT) calculations, we have investigated the effect of local lattice distortions and the effect of local chemical environments on the energy of solution of $\mathrm{C}$ interstitial atoms in tetrahedral and octahedral position in bcc HfNbTiVZr.

\section{METHODS}

\section{A. Interstitial volumes}

There are 6 octahedral sites and 12 tetrahedral sites per unit cell in the bcc structure. Octahedral sites are located at the 

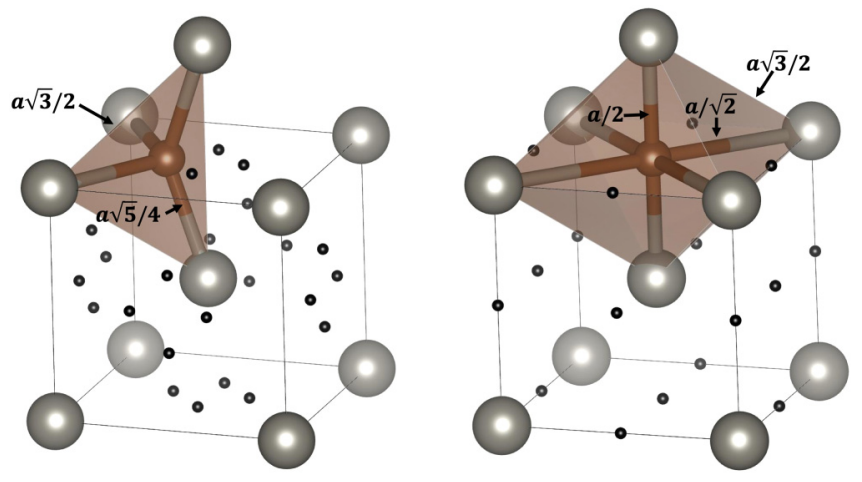

FIG. 1. Tetrahedral and octahedral interstitial sites in the bcc structure.

center of the faces and mid-points of the edges of the unit cell. Octahedral sites are sixfold coordinated, and the octahedron is distorted with two atoms closer to the center than the other four atoms. Tetrahedral sites are located between the center of the faces and mid points of each edge. Figure 1 shows the tetrahedral and octahedral sites in the bcc structure and the nearest neighbor polyhedra.

Using a purely geometric approach, one could speculate that the best position to place an interstitial atom is the one with the most available free space. In a perfect bcc structure, the hard atom sphere model gives an atomic solute radius of $0.154 r_{\mathrm{A}}$ for the octahedral site and $0.291 r_{\mathrm{A}}$ for the tetrahedral site, where $r_{\mathrm{A}}$ is the atomic radius of the constituent atoms. In the HEA case, the multiple atomic sizes of the alloy's components and the different local environments make the assessment of the void's spaces available for the dissolution of an interstitial atom into a nontrivial task. In this work, we estimate the available volume using the so-called radical Voronoi tessellation $[25,26]$. In this method atomic volumes take the form of irregular polyhedra with faces built from the planes which are perpendicular bisectors between a particle and its neighbors. The radical Voronoi tessellation is a generalization of the conventional Voronoi polyhedra procedure to examine multicomponent compounds. The resulting radical plane polyhedra depend on the relative sizes of the atoms, forming an assembly of polyhedra which completely fill space. The radical tessellation includes all points whose tangential distance to the surface of the sphere is smaller than the tangential distance to the other spherical surfaces.

In a HEAs the nearest neighbor polyhedra around the interstices are distorted due to the relaxation effects caused by the presence of the multiple components $[6,27,28]$. We have tested different positions inside the nearest neighbor polyhedral to determine the most suitable candidate position to place an interstitial atom. The radical Voronoi volume for the centroid and for the midpoint of the vector connecting the centroid to the nearest neighbors is calculated, and the position with the largest volume is selected. The probe positions are shown in Fig. 2. We have used the voro++ library for the Voronoi volume calculations [29].

\section{B. DFT calculations}

The DFT calculations are performed using the projector augmented wave method [30] as implemented in the

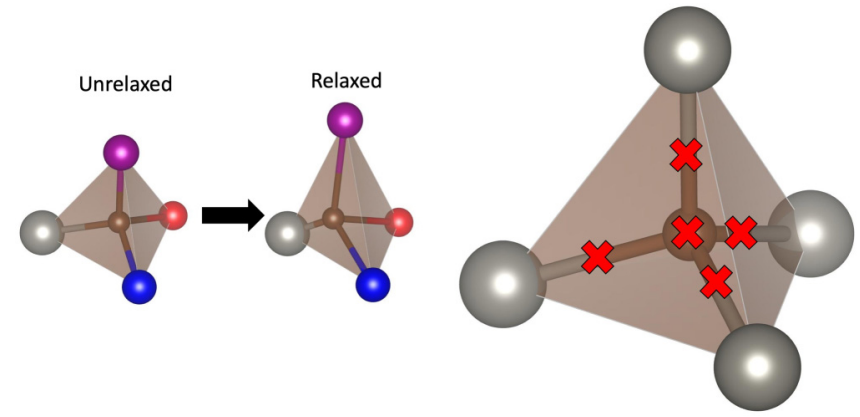

FIG. 2. Schematic comparison of the perfect and distorted nearest neighbor polyhedra, and the positions probed to find the most suitable position to place an interstitial atom.

Vienna Ab-initio Simulation Package (VASP) [31,32] with the Perdew-Burke-Ernzenhof generalized gradient approximation [33] to model the exchange correlation effects. All calculations are performed using a $420 \mathrm{eV}$ kinetic-energy cutoff, with a convergence criterion on forces of $0.01 \mathrm{eV} / \AA$ for structural relaxations. We use a $3 \times 4 \times 5$ HEA supercell with 120 atoms, which allows for an equiatomic composition. The supercell is created using the special quasirandom structure method (SQS) [34] and contains 720 tetrahedral positions and 360 octahedral positions. We have inserted one carbon interstitial into the relaxed HEA supercell for each of the obtained largest available volume positions of the tetrahedral and octahedral interstitial sites and further relaxed the volume, shape, and atom positions of the system.

\section{Solution energies of interstitial atoms}

The solution energies $\Delta E_{\mathrm{sol}}$ of $\mathrm{C}$ atoms are calculated as

$$
\Delta E_{\mathrm{sol}}=E(\text { alloy }+C)-[E(\text { alloy })+E(C)],
$$

where $E($ alloy $+C$ ) and $E$ (alloy) are the energies of the alloys with and without one $\mathrm{C}$ atom. $E(C)$ is the energy of graphite per atom. Due to the inadequate description of van der Waals interactions of the generalized gradient approximation, there is a large overestimation of the energy difference between graphite and diamond $[25,35,36]$. Experimentally it has been found that the enthalpy of graphite is $0.019 \mathrm{eV}$ lower than the diamond enthalpy [37]. To obtain $E(C)$, we calculate the energy of diamond and shift it by applying the experimental difference value.

\section{RESULTS}

\section{A. HfNbTiVZr without carbon}

The size mismatch and different element interactions give rise to considerable distortions in the relaxed system. The relaxation of the carbon free HEA HfNbTiVZr yields a lattice constant of $3.36 \AA$. Fazakas et al. reported a $3.40 \AA$ lattice constant determined from theoretical calculations [9]. The experimental values of the lattice constant are 3.3659(2) $\AA$ [5], 3.36(5) [10], 3.385 [9], and 3.36466(9) $\AA$ [6]. In the relaxed structure, we find an average displacement from ideal bcc positions of $0.27 \AA$ with a standard deviation of $0.13 \AA$. Table I presents the average of the displacements per atomic 
TABLE I. Average displacement per atomic species between ideal and relaxed HfNbTiVZr.

\begin{tabular}{lc}
\hline \hline & Average (̊) \\
\hline $\mathrm{Hf}$ & $0.25(15)$ \\
$\mathrm{Nb}$ & $0.23(9)$ \\
$\mathrm{Ti}$ & $0.30(12)$ \\
$\mathrm{V}$ & $0.31(10)$ \\
$\mathrm{Zr}$ & $0.27(15)$ \\
\hline \hline
\end{tabular}

species. All atomic species display large displacements from their ideal bcc positions, but no particular atomic species' behavior can be discerned due to the large standard deviations. In the relaxed structure, the different local chemical environments generate a distribution of interstitial volumes. In Fig. 3 we have plotted the volumes for an interstitial carbon atom in tetrahedral and octahedral sites using our largest available volume approach with the radical Voronoi tessellation. We have obtained the pair distribution function of the relaxed HfNbTiVZr without carbon and find a very good agreement with the experimental neutron total scattering results [6] as shown in Fig. 4. This validates our usage of a SQS structure modeling the HEA as a fully random alloy. There is a small shift of $0.09 \AA$ in the nearest neighbor peak between calculations and the experimental data that might originate from lattice vibrations not included in the calculations or a small amount of short-range order in the experiments.

\section{B. Energy of solution}

The initial energies of supercells with interstitial carbon placed in the carbon-free relaxed HEA and the final energies after relaxing the supercells with the carbon atom for both tetrahedral and octahedral positions are shown in Fig. 5. The $x$ axis in both Fig. 5 plots has been sorted with respect to initial interstitial void volume in ascending order. For the initial case, it is possible to discern between the behavior of the tetrahedral and octahedral cases. In particular, the

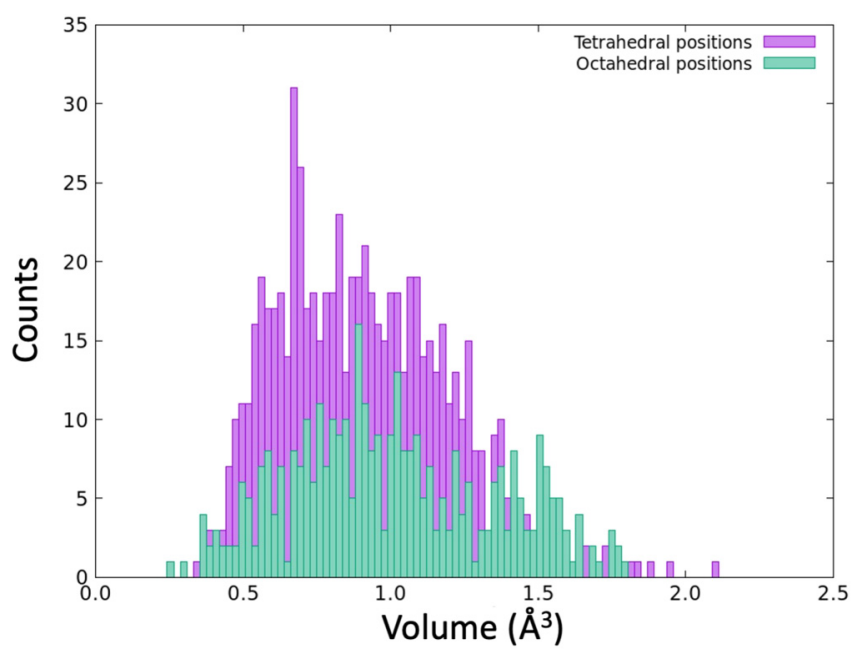

FIG. 3. Radical Voronoi volume distribution of tetrahedral and octahedral sites in the relaxed HEA.

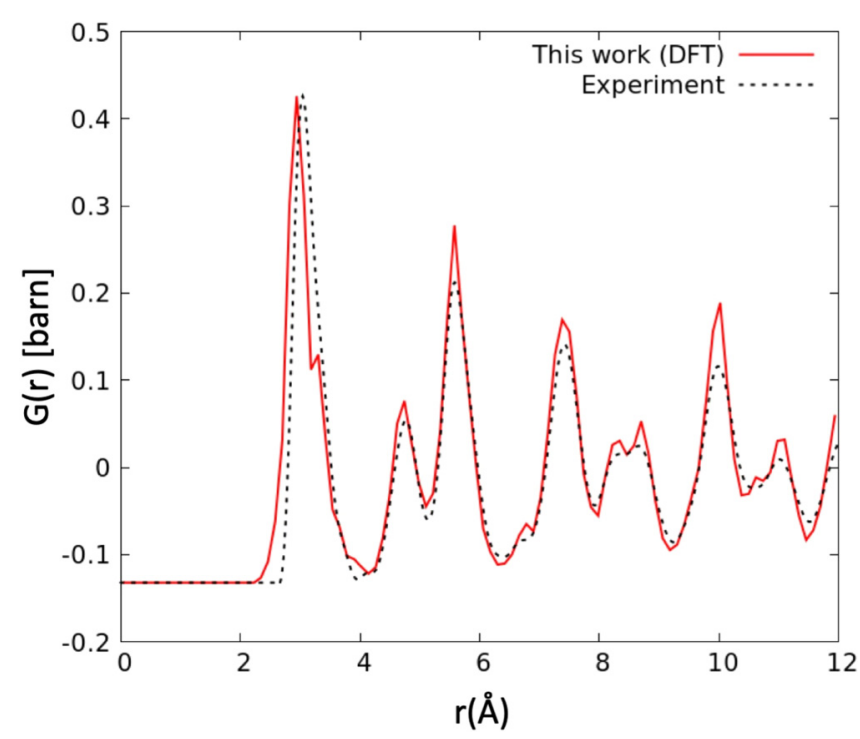

FIG. 4. Pair distribution function of the DFT relaxed HfNbTiVZr and compared with the experimental neutron total scattering results in Ref. [6]. The function from DFT is scaled so that the baseline and the first peak are at the same level as in the experimental function.

octahedral sites display a large range of initial energies, with some octahedral sites having considerably higher and lower energies than the tetrahedral sites. For the initial case, there is a correlation between energy and volume, with larger volumes having lower energies for both the tetrahedral and octahedral positions. Figure 5(b) shows the final energy after relaxation. In this case, there is no clear correlation between the energy and the interstitial volume, and the energies of tetrahedral and octahedral positions are not clearly distinguishable. This is because during relaxation, the carbon atoms initially put on tetrahedral sites diffuse to octahedral sites or interstitial positions in between octahedral and tetrahedral sites. The diffusion of $\mathrm{C}$ could be corroborated by visualizing the atomic positions, but to assess the large number of cases we have employed the Steinhardt bond-orientational parameters [38] and used Pyscal [39] to calculate them (see details in the Supplemental Material [40]). For the octahedral sites, the range in energy values has reduced, from $\sim 20 \mathrm{eV}$ in the initial case to $\sim 2 \mathrm{eV}$ in the final case. This indicates that some initial positions were highly unfavorable, while the most favorable positions were not strongly affected by relaxations. All the carbon interstitials put at octahedral sites stay there. Due to this result, for the remainder of the paper we only show results for carbon in octahedral positions.

Figure 6 shows the distribution of $\Delta E_{\mathrm{sol}}$ of carbon atoms at octahedral positions calculated according to Eq. (1), with an average value of $-1.01 \mathrm{eV}$ and standard deviation $0.31 \mathrm{eV}$. In comparison, we find that a carbon interstitial in an octahedral site in pure bcc $\mathrm{Nb}$ and pure bcc $\mathrm{V}$ has a solution energy of $-0.67 \mathrm{eV}$ and $-0.74 \mathrm{eV}$, respectively, which may indicate that the multicomponent alloying favors $\mathrm{C}$ dissolution in the HEA. Moreover, the lowest solution energy in the HEA with a value of $-1.56 \mathrm{eV}$ (corresponding to a local environment comprising of V-Ti-Ti-Nb-Zr-Zr nearest neighbors) is significantly lower than the mean value. The highest energy corresponds 
(a)

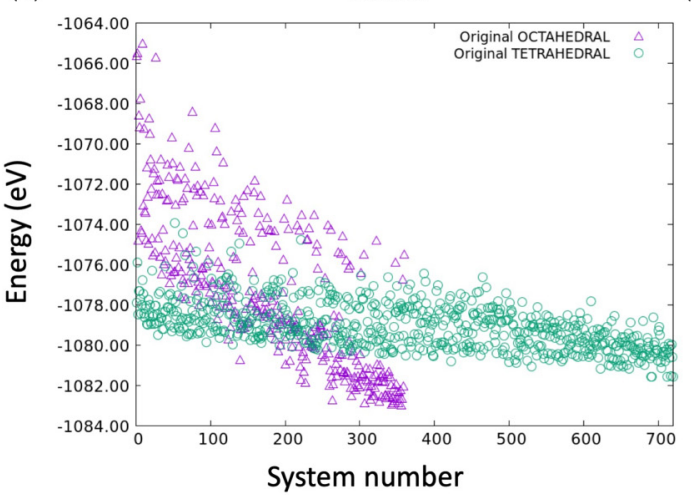

(b)

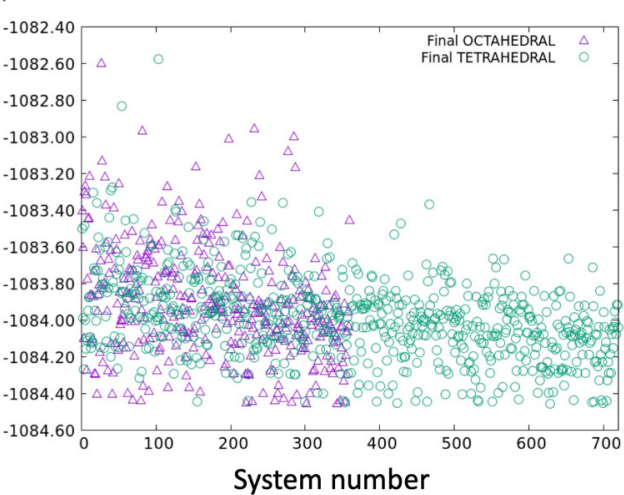

FIG. 5. Total energies of supercells modeling HfNbTiVZr HEA with $\mathrm{C}$ interstitials in octahedral and tetrahedral sites. The initial energy corresponds to the total energy of supercells with interstitial carbon placed in the SQS modeling of a carbon-free relaxed HEA, while the final energy is calculated for the fully relaxed supercell with the carbon atom in the system. The $x$ axis shows each of the interstitial positions, which have been sorted in ascending radical Voronoi volume.

to a $\mathrm{Nb}$ rich local environment with a solution energy of 0.29 $\mathrm{eV}$. This is the only octahedral site with a positive energy of solution. Table II presents the energy of formation of the monocarbides ( $\mathrm{HfC}, \mathrm{NbC}, \mathrm{TiC}, \mathrm{VC}$, and $\mathrm{ZrC}$ ) and the energy of solution of a carbon interstitial in an octahedral site for the pure bcc metals $\mathrm{Nb}$ and $\mathrm{V}$, and hcp $\mathrm{Hf}, \mathrm{Ti}$, and $\mathrm{Zr}$. In the table we have also included the average energies for metal-type$i$-rich $\left(M_{i}\right.$-rich) local environments around carbon interstitial atoms in the HEA. An environment is defined as $M_{i}$ rich when the $M_{i}$ element occupies half or more of the nearest neighbor sites. In all these cases carbon has an octahedral environment. Although the structure of the monocarbides and the pure metals are different, it can be argued that the general trend in $M_{i}$-C bond strength should be similar in both cases. This is observed in Table II, where group 4 (Hf,Ti, Zr) compounds in the monocarbides and reference metals cases have a more negative formation energy suggesting stronger bonds than for those of group $5(\mathrm{Nb}, \mathrm{V})$. However, a different behavior is observed for the HEA where the average value for $\mathrm{V}$ is more negative than $\mathrm{Hf}$ and $\mathrm{Zr}$. Figure 7 shows $\Delta E_{\mathrm{sol}}$ for $M_{i}$-rich lo-

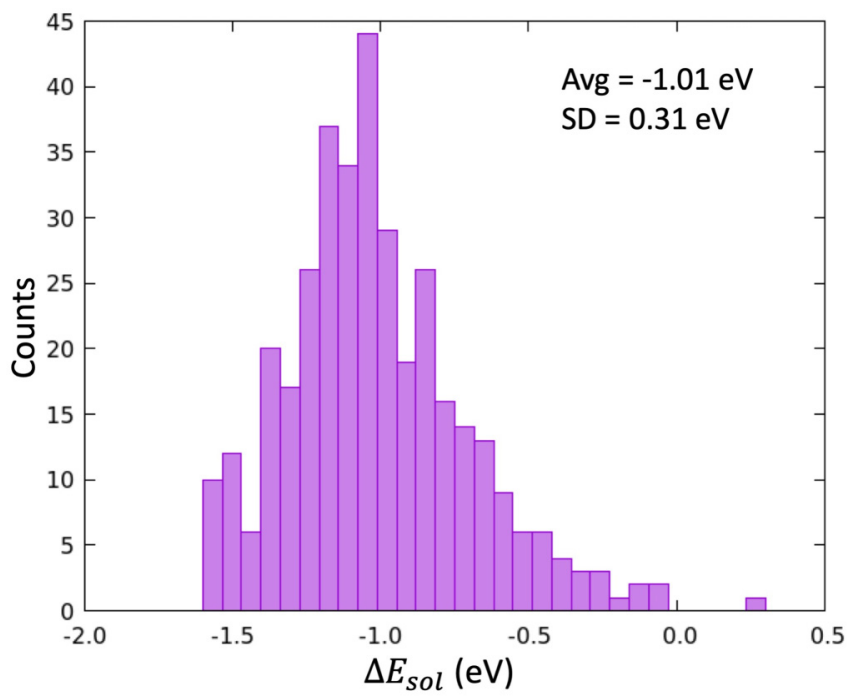

FIG. 6. Energy of solution for octahedral sites. cal environments around carbon interstitial atoms. Among the different $M_{i}$-rich environments, Ti rich is the most favorable, with an average of $-1.23 \mathrm{eV}$ followed by $\mathrm{V}, \mathrm{Hf}, \mathrm{Zr}$, with $\mathrm{Nb}$ being the least favorable. Therefore, it would be more likely to encounter carbon atoms close to Ti-rich local environments of the material. In Fig. 7, we also have included the distribution for "HEA-like" local environments, meaning environments including all five metals with one repeated element type. For the HEA-like case, the lowest energy is $-1.54 \mathrm{eV}$ and highest energy $-0.06 \mathrm{eV}$. A comparison with solution energies of the carbon interstitial in an octahedral site in pure bcc $\mathrm{Nb}$ and pure bcc $\mathrm{V}$ given above shows that the values for the pure metals are 0.18 and $0.29 \mathrm{eV}$ above the mean value of $\mathrm{Nb}$-rich local and V-rich environments, respectively, found in our HEA. The amount of carbon that can be dissolved in the HEA will depend on the number of energetically favorable environments and carbon-carbon interactions. One can imagine that carbon atoms would first occupy the lowest energy sites, in a similar way as proposed for the creation of vacancies in metastable random alloys [41].

\section{Effect of interstitial carbon on local displacements in HfNbTiVZr HEA}

Figure 8 shows the displacements of all the metal atoms in the supercell from their positions in the relaxed carbon

TABLE II. Formation energies per carbon atom of the monocarbides, and energy of solution of interstitial carbon in the dilute limit for the pure metals (bcc structured $\mathrm{Nb}$ and $\mathrm{V}$, and hcp structured $\mathrm{Hf}$, $\mathrm{Ti}$, and $\mathrm{Zr}$ ) and $M_{i}$-rich average in the HEA.

\begin{tabular}{lccc}
\hline \hline & $\begin{array}{c}\text { Monocarbides } \\
(X \mathrm{C}) \\
(\mathrm{eV} / \mathrm{C} \text { atom })\end{array}$ & $\begin{array}{c}\text { Elemental } \\
\text { reference } \\
(\mathrm{eV} / \mathrm{C} \text { atom })\end{array}$ & $\begin{array}{c}\text { HEA } \\
M_{i} \text {-rich average } \\
(\mathrm{eV} / \mathrm{C} \text { atom })\end{array}$ \\
\hline $\mathrm{Hf}$ & -2.01 & -1.84 & -1.01 \\
$\mathrm{Nb}$ & -1.05 & -0.67 & -0.85 \\
$\mathrm{Ti}$ & -1.71 & -1.67 & -1.23 \\
$\mathrm{~V}$ & -0.93 & -0.74 & -1.03 \\
$\mathrm{Zr}$ & -1.83 & -1.73 & -0.94 \\
\hline \hline
\end{tabular}



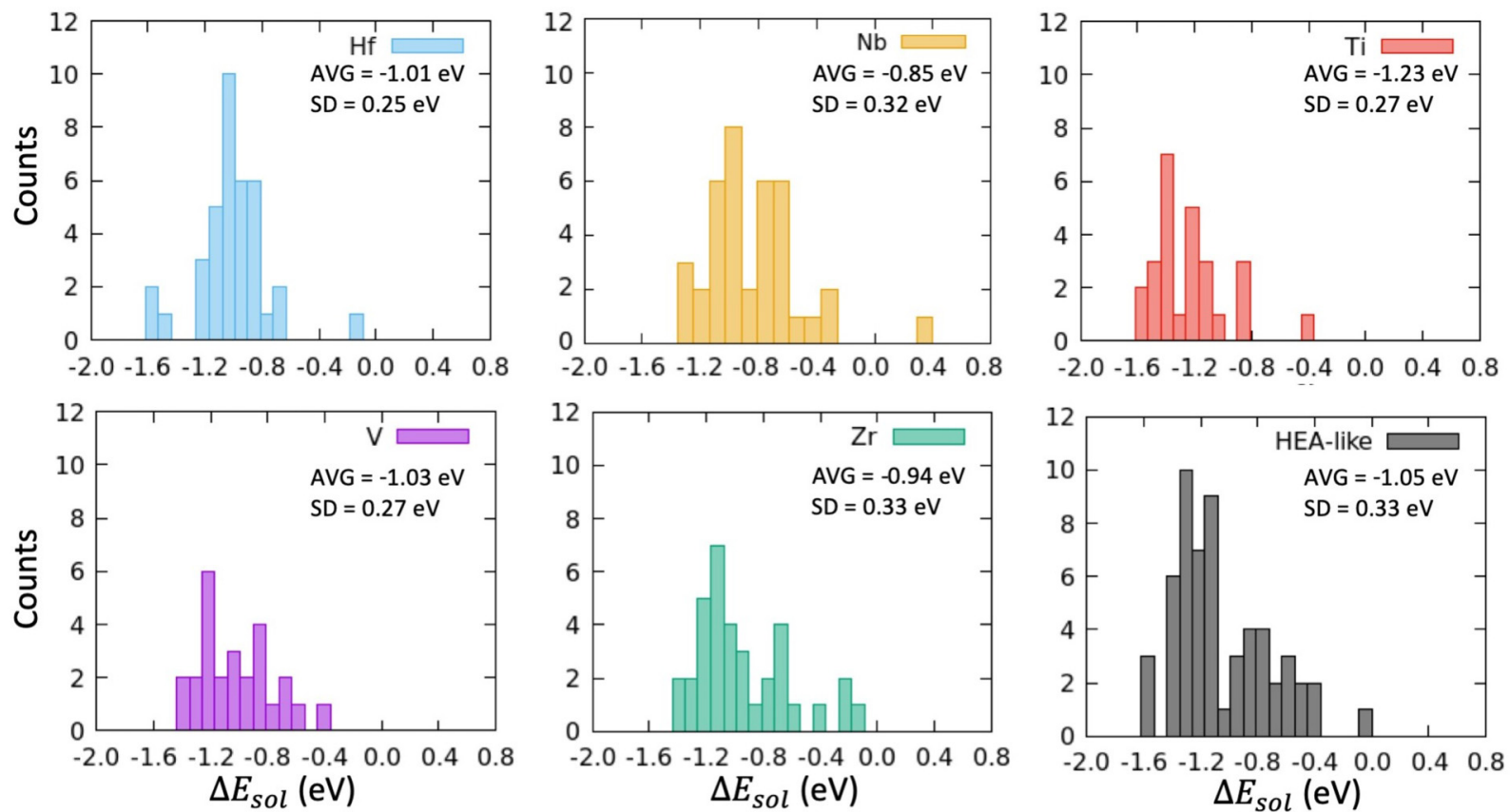

FIG. 7. Distribution of the solution energies of carbon atoms in octahedral sites for different $M_{i}$ rich, as well as the HEA-like local environments.

free HEA when the carbon interstitial is introduced. In this figure we plot the displacement as a function of their distance to the carbon atom, and include the displacements from all the different supercells with interstitial carbon. It is possible to observe the different neighbor shells, and in particular the first neighbor shell is clearly distinguishable. In general, the largest displacements are found in the first coordination sphere, with decreasing magnitude as the positions are farther away from the interstitial. Nonetheless, there are still some large displacements for atoms located at $4 \AA$ from the carbon interstitial, and even the atoms located farthest away from the carbon interstitial present considerable displacements.

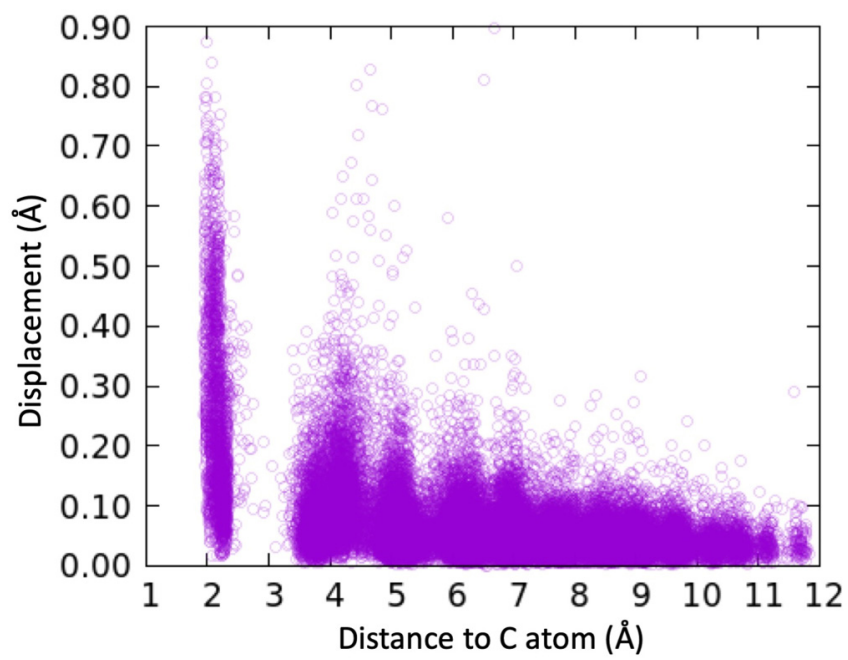

FIG. 8. Atomic displacements of metal atoms upon carbon inclusion as a function of the distance to the carbon interstitial.
For comparison, we have obtained the atom displacements in the case of a carbon atom in bcc $\mathrm{Nb}$ and $\mathrm{V}$, since they are the two constituent elements that have a stable bcc structure at low temperature. In Fig. 9(a), the displacements as a function of distance from the carbon atom are compared with the average displacements in the HEA, and in Fig. 9(b) we show a schematic representation of the displacements of pure $\mathrm{Nb}$, where the displacement vectors have been scaled up for clarity. Figure 9(b) shows that the largest displacements in the pure elements correspond to the atoms in the short axis of the nearest neighbor octahedron, which are pushed away from the carbon interstitial, while the other four nearest neighbors are pulled towards it. In the case of the HEA, it is not possible to discern between these two types of nearest neighbor atoms due to the wide range of nearest neighbor distances. Thus, the average displacement of the first shell in HfNbTiVZr is lower in magnitude and has a large error bar. For the pure element, the displacement tends to become 0 with increasing distance, while for the HEA, even the positions at the greatest distance from $\mathrm{C}$ that is possible to investigate with our supercell have a finite displacement.

\section{Local environment and $\Delta E_{\text {sol }}$}

To investigate the effect of the local chemical environment on the solution energies, we have studied its correlation with volume and local valence electron concentration (VEC). Figure 10 presents the plotted $\Delta E_{\mathrm{sol}}$ versus the nearest neighbor polyhedral volume, the Voronoi volume, the radical Voronoi volume, and the VEC. The VEC has showed a good correlation with the energy of solution in previous studies [24,42]. The VEC is computed as the average of valence electron numbers over the atoms in the first nearest neighbor shell of an interstitial carbon atom, with four valence electrons 

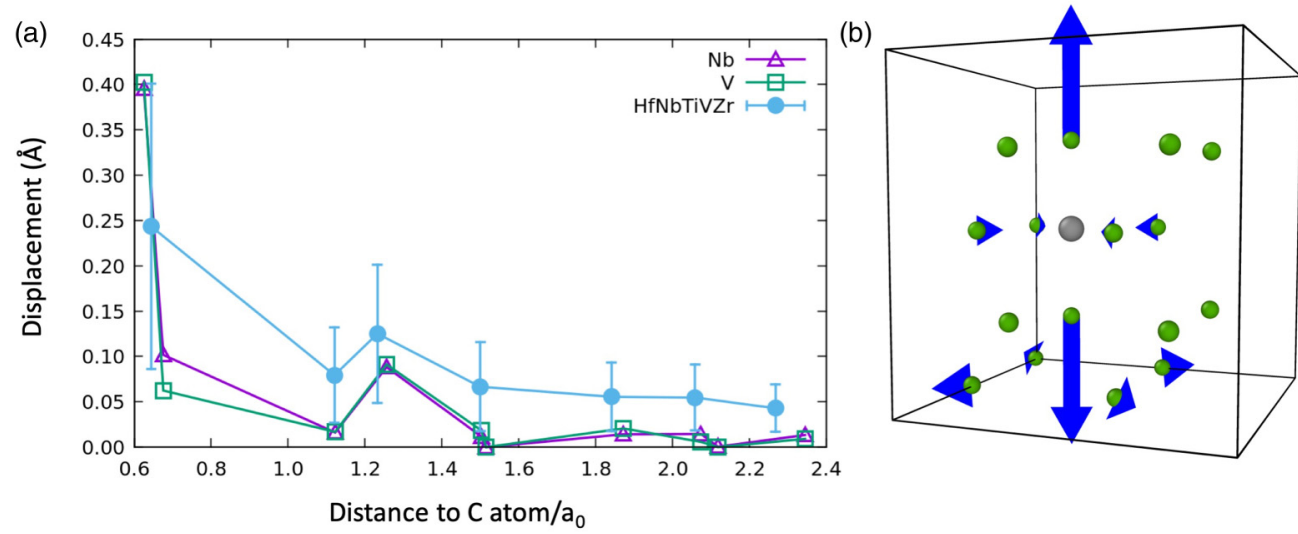

FIG. 9. (a) Comparison of displacements of metal atoms in pure Nb, V and average displacement in HfNbTiVZr as a function of distance to the carbon interstitial. (b) Schematic illustration of atomic displacements in bcc $\mathrm{Nb}$.

for $\mathrm{Hf}$, Ti, and $\mathrm{Zr}$, and five for $\mathrm{Nb}$ and $\mathrm{V}$. For HfNbTiVZr the similar valence electrons for the constituent elements make the VEC a bad descriptor since it is unable to distinguish the different chemical environments. However, among these properties the Voronoi volume shows the best correlation with the solution energies.

Finally, the local chemical environment of carbon atoms in octahedral sites has been evaluated with a local cluster expansion method $[43,44]$. We have tried two approaches for the cluster expansion. In the first one, only the on-site interactions of the number of first nearest neighbors of each element type have been used. In the second approach an effective interaction for the Voronoi volume has been added to the first approach. The expressions for these two approaches are given in Eqs. (2) and (3), respectively. Both approaches fail to describe the individual values of the solution energies.
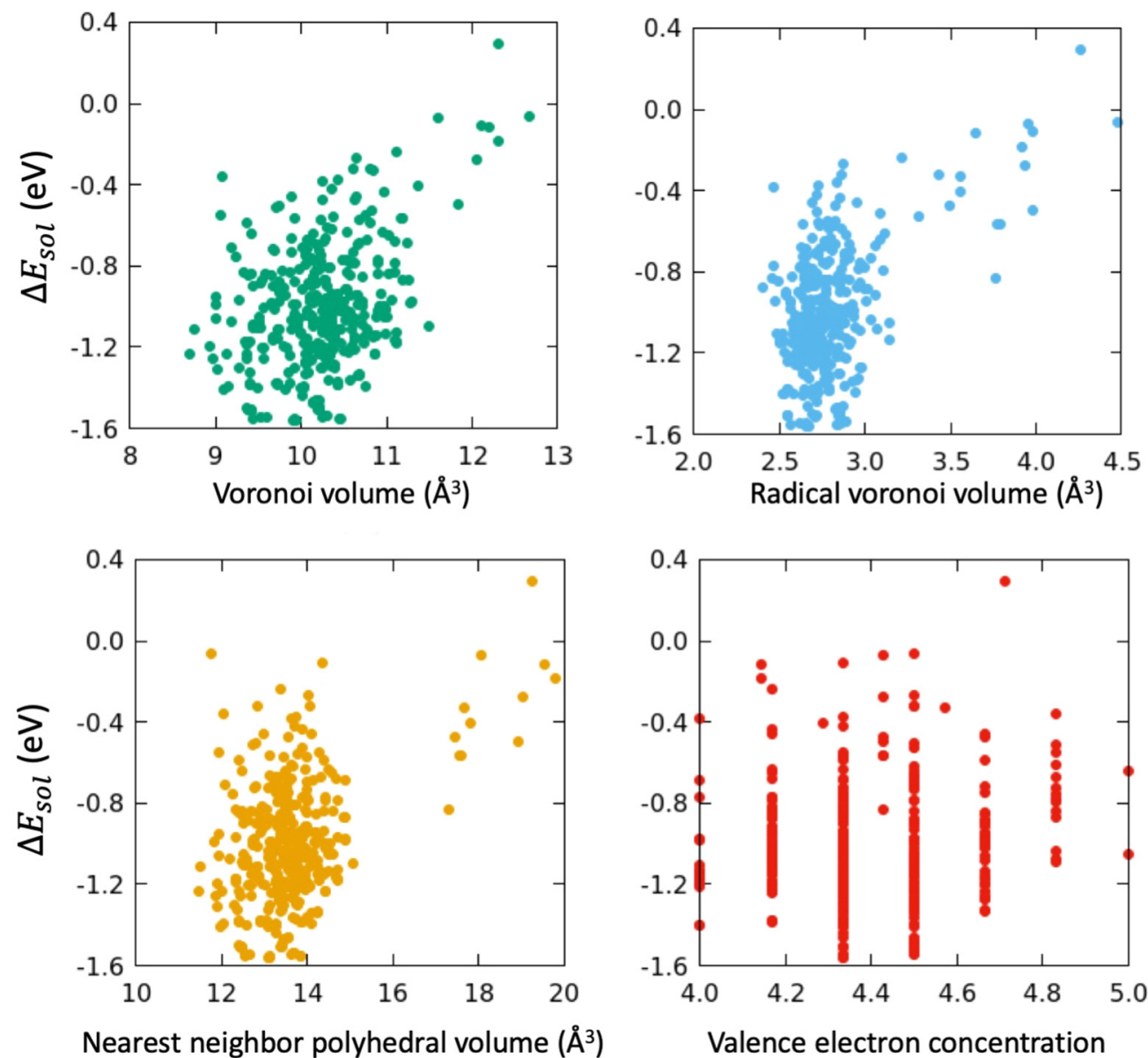

FIG. 10. $\Delta E_{\mathrm{sol}}$ vs Voronoi volume, radical Voronoi volume, nearest neighbor polyhedral volume, and valence electron concentration. 


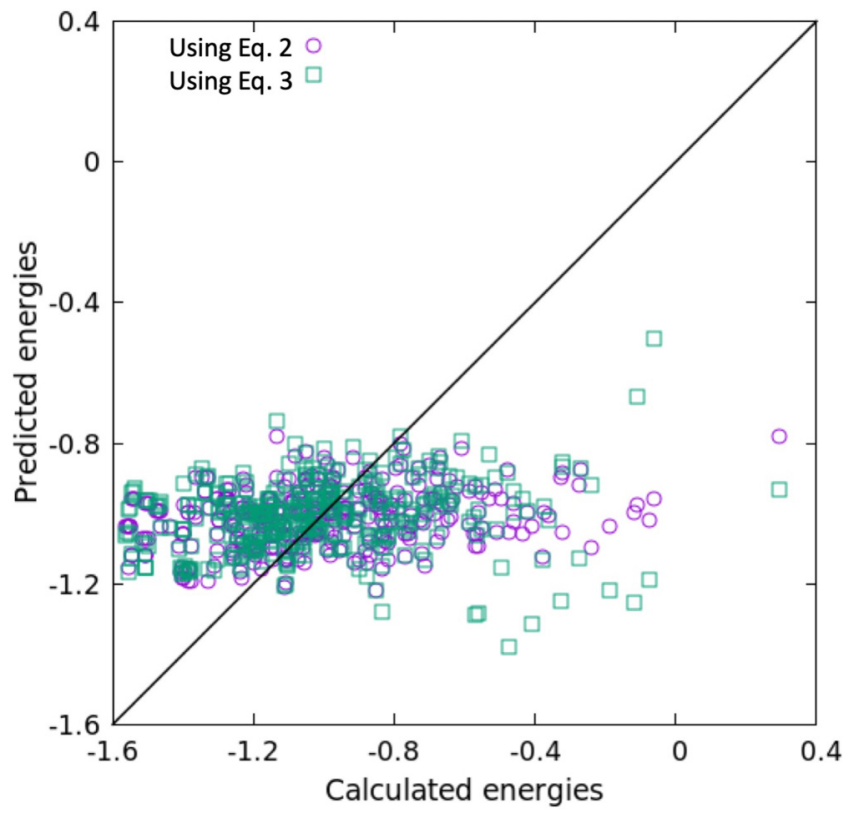

FIG. 11. Calculated vs predicted energies using on-site interactions obtained by a local cluster expansion.

In Fig. 11 we have plotted the comparison of the calculated energies using DFT and the predicted energies. The failure of these simplistic approaches is an indication that the energy of solution has a complex dependence on chemical environment. It is needed to account for interactions well beyond the first neighbor shell and possibly the particular configurations of metals around carbon, as was found for $\mathrm{Ti}$ and $\mathrm{Al}$ around $\mathrm{N}$ in Ref. [45]. This finding is in line with the finding of long-range induced structural relaxations in Figs. 8 and 9,

$$
\begin{gathered}
E_{\mathrm{Sol}} \cong \varepsilon_{\mathrm{Hf}} n_{\mathrm{Hf}}+\varepsilon_{\mathrm{Nb}} n_{\mathrm{Nb}}+\varepsilon_{\mathrm{Ti}} n_{\mathrm{Ti}}+\varepsilon_{\mathrm{V}} n_{\mathrm{V}}+\varepsilon_{\mathrm{Zr}} n_{\mathrm{Zr}} \\
E_{\mathrm{Sol}} \cong \varepsilon_{\mathrm{Hf}} n_{\mathrm{Hf}}+\varepsilon_{\mathrm{Nb}} n_{\mathrm{Nb}}+\varepsilon_{\mathrm{Ti}} n_{\mathrm{Ti}}+\varepsilon_{\mathrm{V}} n_{\mathrm{V}}+\varepsilon_{\mathrm{Zr}} n_{\mathrm{Zr}}+\varepsilon_{\mathrm{Vol}} V_{i} .
\end{gathered}
$$

\section{CONCLUSION}

In this work we have calculated from first principles the energy and structural effects of solution of dilute carbon interstitials in HfNbTiVZr HEA and assessed the impact of local chemical environment on these energies by probing 720 tetrahedral sites and 360 octahedral sites. An excellent agreement between the pair distribution function from the SQS supercell and that from experimental neutron total scattering results has been obtained. We found that carbon atoms initially placed in tetrahedral positions are unstable and move to octahedral or other interstitial positions after relaxation. The solution energies of $\mathrm{C}$ in HfNbTiVZr with respect to HEAs and graphite display a broad distribution, with an average value of $-1.01 \mathrm{eV}$ and a standard deviation $0.31 \mathrm{eV}$. Both the average value and minimum value of $-1.56 \mathrm{eV}$ are significantly lower than the calculated solution energies of $\mathrm{C}$ in pure bcc $\mathrm{Nb}$ and $\mathrm{V}$. The energies of solution were negative for all octahedral sites except one with $\mathrm{Nb}$-rich local environment. Among all environments that are rich in a single metal type, Ti rich presents the most favorable sites for carbon interstitials. The introduction of carbon interstitials produces large distortions in the system that extend beyond the first and second nearest neighbors shells. The wide range of displacements found in the nearest neighbor shell, and the failure of the simple local cluster expansion approaches indicate that interaction with atoms beyond the first nearest neighbor shell are needed to understand the complex distribution of local solution energetics for this material system.

\section{ACKNOWLEDGMENTS}

This work was supported by the Swedish Research Council (VR) Project No. 2018-04834. Support from the Swedish Government Strategic Research Area in Materials Science on Functional Materials at Linköping University, Faculty Grant SFOMatLiU No. 200900971 and from the Knut and Alice Wallenberg Foundation (Wallenberg Scholar Grant No. KAW2018.0194) is gratefully acknowledged. B.A. acknowledges financial support from the Swedish Foundation for Strategic Research through the Future Research Leaders 6 program, FFL 15-0290 and from the Swedish Research Council (VR) through Grant No. 2019-05403, Theoretical analysis of the results of electronic structure calculations was funded by RFBR, Project No. 20-02-00178. Calculations were performed using supercomputer resources provided by the Swedish National Infrastructure for Computing (SNIC) at the National Supercomputer Center (NSC). M.M.N., M.H.S., and B.C.H. acknowledge funding by the NordForsk Nordic Neutron Science Programme through the functional hydrides (FunHy) project (Grant No. 81942).
[1] O. N. Senkov, G. B. Wilks, J. M. Scott, and D. B. Miracle, Mechanical properties of $\mathrm{Nb}_{25} \mathrm{Mo}_{25} \mathrm{Ta}_{25} \mathrm{~W}_{25}$ and $\mathrm{V}_{20} \mathrm{Nb}_{20} \mathrm{Mo}_{20} \mathrm{Ta}_{20} \mathrm{~W}_{20}$ refractory high entropy alloys, Intermetallics 19, 698 (2011).

[2] M. M. Nygård, G. Ek, D. Karlsson, M. Sahlberg, M. H. Sørby, and B. C. Hauback, Hydrogen storage in high-entropy alloys with varying degree of local lattice strain, Int. J. Hydrog. 44, 29140 (2019).

[3] M. M. Nygård, G. Ek, D. Karlsson, M. H. Sørby, M. Sahlberg, and B. C. Hauback, Counting electrons-A new approach to tailor the hydrogen sorption properties of high-entropy alloys, Acta Mater. 175, 121 (2019).

[4] M. Sahlberg, D. Karlsson, C. Zlotea, and U. Jansson, Superior hydrogen storage in high entropy alloys, Sci. Rep. 6, 36770 (2016).

[5] D. Karlsson, G. Ek, J. Cedervall, C. Zlotea, K. T. Møller, T. C. Hansen, J. Bednarcik, M. Paskevicius, M. H. Sørby, and T. R. Jensen, Structure and hydrogenation properties of a HfNbTiVZr high-entropy alloy, Inorg. Chem. 57, 2103 (2018). 
[6] M. M. Nygård, W. A. Slawinski, G. Ek, M. H. Sørby, M. Sahlberg, D. A. Keen, and B. C. Hauback, Local order in high-entropy alloys and associated deuterides - a total scattering and Reverse Monte Carlo study, Acta Mater. 199, 504 (2020).

[7] N. Ishizu and J. Kitagawa, New high-entropy alloy superconductor $\mathrm{Hf}_{21} \mathrm{Nb}_{25} \mathrm{Ti}_{15} \mathrm{~V}_{15} \mathrm{Zr}_{24}$, Results Phys. 13, 102275 (2019).

[8] V. Pacheco, G. Lindwall, D. Karlsson, J. Cedervall, S. Fritze, G. Ek, P. Berastegui, M. Sahlberg, and U. Jansson, Thermal stability of the HfNbTiVZr high-entropy alloy, lnorg. Chem. 58, 811 (2018).

[9] E. Fazakas, V. Zadorozhnyy, L. Varga, A. Inoue, D. LouzguineLuzgin, F. Tian, and L. Vitos, Experimental and theoretical study of $\mathrm{Ti}_{20} \mathrm{Zr}_{20} \mathrm{Hf}_{20} \mathrm{Nb}_{20} \mathrm{X}_{20}(\mathrm{X}=\mathrm{V}$ or $\mathrm{Cr}$ ) refractory highentropy alloys, Int. J. Refract. Met. Hard Mater. 47, 131 (2014).

[10] M. Feuerbacher, T. Lienig, and C. Thomas, A single-phase bcc high-entropy alloy in the refractory $\mathrm{Zr}-\mathrm{Nb}-\mathrm{Ti}-\mathrm{V}-\mathrm{Hf}$ system, Scr. Mater. 152, 40 (2018).

[11] M. Beyramali Kivy, C. S. Kriewall, and M. Asle Zaeem, Formation of chromium-iron carbide by carbon diffusion in AlXCoCrFeNiCu high-entropy alloys, Mater. Res. Lett. 6, 321 (2018).

[12] J. Chen, Z. Yao, X. Wang, Y. Lu, X. Wang, Y. Liu, and X. Fan, Effect of $\mathrm{C}$ content on microstructure and tensile properties of as-cast $\mathrm{CoCrFeMnNi}$ high entropy alloy, Mater. Chem. Phys. 210, 136 (2018).

[13] L. B. Chen, R. Wei, K. Tang, J. Zhang, F. Jiang, L. He, and J. Sun, Heavy carbon alloyed FCC-structured high entropy alloy with excellent combination of strength and ductility, Mater. Sci. Eng. A 716, 150 (2018).

[14] J. T. Fan, L. J. Zhang, P. F. Yu, M. D. Zhang, D. J. Liu, Z. Zhou, P. Cui, M. Z. Ma, Q. Jing, G. Li, and R. P. Liu, Improved the microstructure and mechanical properties of AlFeCoNi highentropy alloy by carbon addition, Mater. Sci. Eng. A 728, 30 (2018).

[15] Z. Li, Interstitial equiatomic CoCrFeMnNi high-entropy alloys: Carbon content, microstructure, and compositional homogeneity effects on deformation behavior, Acta Mater. 164, 400 (2019).

[16] Z. Li, C. C. Tasan, H. Springer, B. Gault, and D. Raabe, Interstitial atoms enable joint twinning and transformation induced plasticity in strong and ductile high-entropy alloys, Sci. Rep. 7, 40704 (2017).

[17] H. Luo, Z. Li, W. Lu, D. Ponge, and D. Raabe, Hydrogen embrittlement of an interstitial equimolar high-entropy alloy, Corros. Sci. 136, 403 (2018).

[18] Y. Y. Shang, Y. Wu, J. Y. He, X. Y. Zhu, S. F. Liu, H. L. Huang, K. An, Y. Chen, S. H. Jiang, H. Wang, X. J. Liu, and Z. P. Lu, Solving the strength-ductility tradeoff in the mediumentropy $\mathrm{NiCoCr}$ alloy via interstitial strengthening of carbon, Intermetallics 106, 77 (2019).

[19] Z. Wang and I. Baker, Interstitial strengthening of a f.c.c. FeNiMnAlCr high entropy alloy, Mater. Lett. 180, 153 (2016).

[20] Z. Wang, I. Baker, Z. Cai, S. Chen, J. D. Poplawsky, and W. Guo, The effect of interstitial carbon on the mechanical properties and dislocation substructure evolution in $\mathrm{Fe}_{40.4} \mathrm{Ni}_{11.3} \mathrm{Mn}_{34.8} \mathrm{Al}_{7.5} \mathrm{Cr}_{6}$ high entropy alloys, Acta Mater. 120, 228 (2016)
[21] Z. Wang, I. Baker, W. Guo, and J. D. Poplawsky, The effect of carbon on the microstructures, mechanical properties, and deformation mechanisms of thermo-mechanically treated $\mathrm{Fe}_{40.4} \mathrm{Ni}_{11.3} \mathrm{Mn}_{34.8} \mathrm{Al}_{7.5} \mathrm{Cr}_{6}$ high entropy alloys, Acta Mater. 126, 346 (2017).

[22] M. Wu, Z. Li, B. Gault, P. Munroe, and I. Baker, The effects of carbon on the phase stability and mechanical properties of heat-treated FeNiMnCrAl high entropy alloys, Mater. Sci. Eng. A 748, 59 (2019).

[23] Z. Wu, C. M. Parish, and H. Bei, Nano-twin mediated plasticity in carbon-containing FeNiCoCrMn high entropy alloys, J. Alloys Compd. 647, 815 (2015).

[24] Y. Ikeda, F. Körmann, I. Tanaka, and J. Neugebauer, Impact of chemical fluctuations on stacking fault energies of $\mathrm{CrCoNi}$ and $\mathrm{CrMnFeCoNi}$ high entropy alloys from first principles, Entropy 20, 655 (2018)

[25] C. Domain, C. S. Becquart, and J. Foct, Ab initio study of foreign interstitial atom $(\mathrm{C}, \mathrm{N})$ interactions with intrinsic point defects in $\alpha$-Fe, Phys. Rev. B 69, 144112 (2004).

[26] V. Rosato, Comparative behavior of carbon in b.c.c. and f.c.c. iron, Acta Metall. 37, 2759 (1989).

[27] L. Owen, E. Pickering, H. Playford, H. Stone, M. Tucker, and N. Jones, An assessment of the lattice strain in the $\mathrm{CrMnFeCoNi}$ high-entropy alloy, Acta Mater. 122, 11 (2017).

[28] Y. Tong, S. Zhao, H. Bei, T. Egami, Y. Zhang, and F. Zhang, Severe local lattice distortion in $\mathrm{Zr}$-and/or Hf-containing refractory multi-principal element alloys, Acta Mater. 183, 172 (2020).

[29] C. Rycroft, Voro++: A three-dimensional Voronoi cell library in $\mathrm{C}++$, Report No. LBNL-1432E, Lawrence Berkeley National Laboratory (LBNL, Berkeley, CA, 2009).

[30] P. E. Blöchl, Projector augmented-wave method, Phys. Rev. B 50, 17953 (1994).

[31] G. Kresse and J. Furthmüller, Efficiency of ab-initio total energy calculations for metals and semiconductors using a plane-wave basis set, Comput. Mater. Sci. 6, 15 (1996).

[32] G. Kresse and J. Furthmüller, Efficient iterative schemes for ab initio total-energy calculations using a plane-wave basis set, Phys. Rev. B 54, 11169 (1996).

[33] J. P. Perdew, K. Burke, and M. Ernzerhof, Generalized Gradient Approximation Made Simple, Phys. Rev. Lett. 77, 3865 (1996).

[34] A. Zunger, S.-H. Wei, L. G. Ferreira, and J. E. Bernard, Special Quasirandom Structures, Phys. Rev. Lett. 65, 353 (1990).

[35] A. Dick, F. Körmann, T. Hickel, and J. Neugebauer, Ab initio based determination of thermodynamic properties of cementite including vibronic, magnetic, and electronic excitations, Phys. Rev. B 84, 125101 (2011).

[36] B. Hallstedt, D. Djurovic, J. von Appen, R. Dronskowski, A. Dick, F. Körmann, T. Hickel, and J. Neugebauer, Thermodynamic properties of cementite (Fe3C), Calphad 34, 129 (2010).

[37] H. W. Day, A revised diamond-graphite transition curve, Am. Mineral. 97, 52 (2012).

[38] P. J. Steinhardt, D. R. Nelson, and M. Ronchetti, Bondorientational order in liquids and glasses, Phys. Rev. B 28, 784 (1983).

[39] S. Menon, G. Leines, and J. Rogal, Pyscal: A python module for structural analysis of atomic environments, J. Open Source Softw. 4, 1824 (2019). 
[40] See Supplemental Material at http://link.aps.org/supplemental/ 10.1103/PhysRevMaterials.4.123601 for details on the Steinhardt bond-orientational parameter calculations.

[41] F. Tasnádi, A. V. Lugovskoy, M. Odén, and I. A. Abrikosov, Non-equilibrium vacancy formation energies in metastable alloys-A case study of $\mathrm{Ti}_{0.5} \mathrm{Al}_{0.5} \mathrm{~N}$, Mater. Des. 114, 484 (2017).

[42] J. von Appen and R. Dronskowski, Carbon-induced ordering in manganese-rich austenite-a density-functional total-energy and chemical-bonding study, Steel Res. Int. 82, 101 (2011).
[43] J. M. Sanchez, F. Ducastelle, and D. Gratias, Generalized cluster description of multicomponent systems, Physica A 128, 334 (1984).

[44] A. Van der Ven and G. Ceder, Vacancies in ordered and disordered binary alloys treated with the cluster expansion, Phys. Rev. B 71, 054102 (2005).

[45] B. Alling, A. V. Ruban, A. Karimi, O. E. Peil, S. I. Simak, L. Hultman, and I. A. Abrikosov, Mixing and decomposition thermodynamics of $\mathrm{C}-\mathrm{Ti}_{1-x} \mathrm{Al}_{x} \mathrm{~N}$ from first-principles calculations, Phys. Rev. B 75, 045123 (2007). 